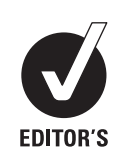

CHOICE

\title{
Effects of Ramadan on physical performance: chronobiological considerations
}

\author{
Jim Waterhouse
}

\section{Correspondence to}

Professor Jim Waterhouse,

3 Cherry Holt Avenue, Heaton

Mersey, Stockport, Cheshire

SK4 3PT, UK;

waterhouseathome@hotmail. com

Accepted 9 March 2010

\section{ABSTRACT}

This article focuses on changes in Ramadan during the course of the waking day in physical performance, mental performance and gastrointestinal function. Possible explanations of the changes are considered by reference to the roles of the body clock and external factors such as the environment and the individual's lifestyle. The effects of these factors upon training schedules and upon changing the times of training are then discussed in terms of physiology and biochemistry. Delaying the time of training until after sunset has advantages and disadvantages, but it is a regimen that is unlikely to be widely accepted in non-Muslim countries. It is clear that the detailed nature of the problems involved and dealing with them in a manner based upon a firm body of knowledge requires far more experimental investigation.

\section{CHANGES IN PERFORMANCE IN RAMADAN}

Ramadan requires Muslims to alter the timing of food and fluid intakes; in addition, their hours of sleep might be curtailed (due to rising earlier and/or retiring later). This combination of factors is likely to affect daytime activities and performance in the mental, physical and social domains. In support of such a prediction, there is evidence of increased incidence of traffic accidents occurring in Ramadan, ${ }^{1}$ and irritability increases. ${ }^{2}{ }^{3}$ Whether these events are related to changes in insulin, blood glucose, low energy levels or mood changes-or some combination-is unclear, but they apply to the population as a whole.

Several studies have investigated performance in individuals undertaking training sessions in sports. Aspects of physical performance are compromised, but the deterioration is often not marked, and other aspects are unaffected. For example, in soccer players, a deterioration in $\mathrm{Vo}_{2}$ max but not sprinting ability or agility, ${ }^{4}$ a fall in aerobic capacity, endurance and jumping ability but no change to sprinting or agility, ${ }^{5}$ a decreased ability to perform exercise at $75 \% \mathrm{Vo}_{2} \max ,{ }^{6}$ no clear changes in speed, power, agility, endurance and dribbling skills, ${ }^{7}$ a marginally reduced (but biologically nonsignificant) decrease in maximal heart rate but no change in perceived exertion, ${ }^{8}$ and a general decrease in fitness and tests of skill (speed, agility, dribbling and endurance) $)^{9}$ have all been reported. In addition, some studies indicated that subjective assessments of performance had been performed less well ${ }^{9}$ or that the exertion required to undertake the training sessions was increased. ${ }^{10} 11$ When deterioration has taken place, it seems to be temporary and reverses soon after the end of Ramadan. ${ }^{4}$

Even if there is deterioration in performance in sports, it is unclear whether there is a systematic decline in physiological variables related to exercise. Ramadan $^{12}$ reported no adverse effects on sedentary subjects exercising at about $70 \% \dot{\mathrm{Vo}}_{2} \max$ under thermoneutral conditions, but there were increases in systolic blood pressure in response to exercise by the end of the month of fasting. Bigard et a ${ }^{13}$ measured maximal voluntary contraction (MVC) of elbow flexion muscles; maximum isometric strength decreased by $10-12 \%$, while muscle endurance at $35 \%$ and $55 \%$ MVC had decreased by $28 \%$ and $22 \%$, respectively, by the end of Ramadan. Siddiqui et all ${ }^{14}$ found no significant changes in vital capacity, forced expiratory volume in $1 \mathrm{~s}$, peak expiratory flow or maximum voluntary ventilation during Ramadan in healthy subjects.

Therefore, it appears that training is compromised to a small extent only, some aspects of training (eg, sprinting and agility) being comparatively immune. However, the individuals in these studies are likely to have been highly motivated and are aware that their performance is being measured-circumstances that will increase the effort made and oppose deterioration. This problem - the subject is aware that measurements are being made-is a general one, and there is no easy way to improve the experimental design by 'blinding' the subject. However, other changes (biochemical, eg, see below) might not be masked by an individual's motivation.

\section{Possible explanations for changes}

The studies described so far do not enable the mechanism(s) by which any decrements in exercise performance during Ramadan to be determined. Falls in performance may be determined by disturbances of the sleep-wake cycle, by changed food and fluid intakes and/or by alterations in circadian rhythms-or, more likely, by an interaction of these factors.

\section{Sleep loss}

Bahammam ${ }^{15}$ investigated the effect of Ramadan on sleep patterns, sleep architecture and daytime sleepiness in eight healthy Muslims. Compared with baseline (non-fasting) days, bedtime was delayed in the first and third weeks of Ramadan, by $1 \mathrm{~h} 18 \mathrm{~min}$ and $1 \mathrm{~h} 36 \mathrm{~min}$, respectively. These delays were coupled with a constant wake-up time, which caused a significant reduction in nocturnal sleep time. This reduction in sleep caused daytime fatigue, but there was also an increase in the amount of sleep taken during the daytime (see also Margolis and Reed ${ }^{16}$ and Roky et a ${ }^{17}$ ), thus reducing the total sleep loss. Bahammam ${ }^{15}$ and Roky et a ${ }^{17}$ examined polysomnographically the nocturnal sleep of Muslims during Ramadan and compared recordings with pre-Ramadan baselines. Their 
main findings were an increase in sleep latency and a modification of sleep architecture. The total sleep time decreased, but the proportion of non-rapid eye movement sleep increased. Within non-REM (rapid eye movement) sleep, there was an increase in stage 2 and a decrease in slow-wave sleep (SWS), these changes being associated with raised rectal temperatures.

Sleep loss produces little direct effect upon muscle activity, but it has an indirect effect upon physical performance via changes in mental performance, coordination and motivation. ${ }^{18}$ Thus, sleep loss results in falls in the level of performance of tasks requiring sensorimotor coordination or cognitive processes, the decrement increasing with amount of sleep lost and importance of the neural component of a task. In addition, there will be a loss of motivation, which can be detrimental in repetitive tasks or those requiring repeated training sessions where the outcome is not immediately realised.

\section{Fasting}

Alertness and mood also will be negatively affected by fasting. Roky et al ${ }^{1}$ concluded that daytime subjective alertness, evaluated by a visual analogue scale (in which subjects scored how alert they felt on a scaled continuum), decreased at 09:00 and 16:00, and showed an increase at 23:00 during Ramadan. They concluded that a reduced energy intake and sleep loss were responsible. A role of decreased food intake was supported by observation of improved mood in the evening after fasting had ended (though increased social activities at this time would have contributed to this improvement). Eating, particularly carbohydrate intake, maintains alertness and mood; ${ }^{19}$ therefore, food and fluid restriction might contribute to negative effects on mood, mental performance and irritability during Ramadan.

The changes in eating and drinking habits are likely to have effects also on body weight, composition and metabolism, on body osmolality and on haematological indices. Several studies have considered these possibilities (see other references in the current issue $e^{46101120-28}$ ). Conclusions from these studies include evidence for dehydration and changed metabolism, with fats becoming a more important energy source.

In summary, changes in sleep habits and food and fluid intakes cause at least some aspects of performance to deteriorate in Ramadan. However, most of these studies have not investigated changes in performance during the course of the day; this is important because 'performance' is not constant throughout the daytime, instead showing rhythmicity, as do other physiological and biochemical variables.

\section{RHYTHMS OF PERFORMANCE}

The body's physiology and biochemistry varies during the course of the $24 \mathrm{~h}$. Humans are naturally geared to be active and seek food in the daytime and to sleep and recuperate at night. These rhythms result from a complex interaction between the environment and the 'body clock.' In order to appreciate how Ramadan might impact on circadian rhythms, an understanding of key features of the body clock is necessary, together with the influence of another factor, time awake.

\section{Body clock}

The suprachiasmatic nuclei (SCN) of the hypothalamus are the site of the body clock. ${ }^{29}$ These clusters of cells are linked to the eyes via the direct retinohypothalamic tract and receive a humoral input from the pineal gland, which secretes the hormone melatonin during the hours of darkness. Melatonin has many effects throughout the body, reviewed in an exercise physiology context by Atkinson et al. ${ }^{30}$ One of melatonin's properties is that it is a potent vasodilator and so promotes heat loss. The evening fall of body temperature is caused by the body clock and the onset of melatonin secretion; the morning rise in body temperature is due to the combined effects of the body clock and an inhibition of melatonin secretion (secretion being suppressed by bright light). Melatonin induces drowsiness through its effect upon body temperature and also via a direct central action.

The rhythmic neural and humoral inputs to the SCN (showing a period of $24 \mathrm{~h}$ ) enable the phase of the body clock to be adjusted to the environment, particularly the light-dark cycle. The mechanism by which such phase changes are achieved is complex, but in essence, bright light in the morning (from about 04:00, the time of the temperature minimum, until 10:00 h) advances the clock, and bright light in the evening (from about 22:00 to 04:00) delays it. Melatonin secretion also affects the phase of the body clock but in the opposite direction to light; in the morning, melatonin delays the body clock and, in the evening, advances it. Since bright light suppresses melatonin secretion (see above), morning light advances the body clock not only directly but also indirectly by suppressing melatonin secretion and so opposing the delay that melatonin would have exerted at this time. Since they adjust the phase of the body clock, rhythms of light and melatonin secretion are known as zeitgebers (German for 'time-givers'), light and melatonin being the main zeitgebers in humans. ${ }^{31}$

There is limited evidence that exercise and mealtimes can act as a weak zeitgeber; exercise produces a weak delay when undertaken in the evening and night and a small advance around noon and in the early afternoon. ${ }^{32}$ Mealtimes might act as a zeitgeber by reinforcing an individual's sleep-wake cycle, and rhythms of social and mental activity might play similar roles. In practice, the main zeitgebers (light-dark cycle and rhythm of melatonin secretion) and the subsidiary ones (rhythms of physical, social and mental activities) produce a 'package' which adjusts the individual's physiology and biochemistry to daytime activity and nocturnal rest.

Outputs from the SCN are transmitted to hypothalamic nuclei that control body temperature and hormone secretion, to the raphe nucleus which is involved in sleep regulation, and to the sympathetic nervous system; as a result, the rhythmic output of the SCN pervades the whole body. For the purposes of the present article, the most important rhythms are those of physical and cognitive activities, of food intake and of digestion and metabolism of the absorbed foodstuffs.

\section{Interpreting circadian rhythms}

An observed circadian rhythm is not produced by the body clock only (the endogenous component of the observed rhythm); another part of the rhythm is due to a direct effect of the individual's environment and sleep-wake cycle (the exogenous component, ${ }^{29}$ the nature of which depends upon the variable under consideration). Thus, core temperature is raised by activity and lowered by the change in posture associated with sleep; melatonin secretion is suppressed by bright light; antidiuretic hormone is increased by standing up and dehydration; growth hormone secretion is stimulated by SWS; gut activity and the secretion of digestive enzymes are stimulated by the presence of food in the gastrointestinal tract; and the secretion of insulin is stimulated by absorption of glucose into the bloodstream.

The demonstration of the existence of these endogenous and exogenous components is illustrated by figure 1. In this figure, the temperature rhythm of eight males is shown when living normally (sleeping from midnight to 08:00) and then when undergoing a 'constant routine.' In this routine, subjects 
are required to stay awake and sedentary (or even lying down) for a period of at least $24 \mathrm{~h}$ in an environment of constant temperature and lighting. Identical meals are given each hour. The rhythm remaining in this protocol cannot be attributed to food intake, the environment or the sleep-wake cycle; therefore, it is termed the endogenous component of the rhythm. The difference between the two curves, due to normal changes in the environment, the sleep-wake cycle and the rhythmic intake of food, is termed the exogenous component.

The relative size of these two components depends upon the variable. For plasma melatonin, the endogenous component is strong (and so it can be used as a marker of the phase of the body clock); for most respiratory and cardiorespiratory variables, it is weak (these variables responding more to the immediate demands of the individual's lifestyle); and for core temperature, the two components are of about equal size. Hormones associated with metabolism vary in the relative importance of their exogenous and endogenous components-a variation that is demonstrated by comparisons of growth hormone (that is mainly exogenous and secreted during sleep; see above), cortisol (which has a high endogenous component, comparable with melatonin) and insulin (which has a marked exogenous component due to plasma glucose levels but is also affected to some extent by the body clock).

In subjects living normally (asleep at night and active in the daytime), the endogenous and exogenous components of a circadian rhythm are in phase (see figure 1), thereby ensuring that the individual's rhythms are fully adjusted to the demands of a diurnal lifestyle. In the morning, the individual becomes progressively more active as core temperature and plasma epinephrine rise, and the environment changes from a relaxing to an alerting one. In the daytime, these variables, the individual's activities and the stimulating nature of the environment reach their peak; metabolism is mainly of glucose with the excess of calorie intake over requirements being stored as lipid in adipose tissue. In the evening, body temperature and epinephrine fall as melatonin levels rise, the environment becomes quieter and more relaxing, and the individual prepares for sleep. During the night, hormones associated with growth and repair are secreted, and the body's energy source is mainly derived from adipose tissue.

An important implication is that the observations of changed rhythms in Ramadan, for example, cannot be interpreted unambiguously as due to a shift in the endogenous component

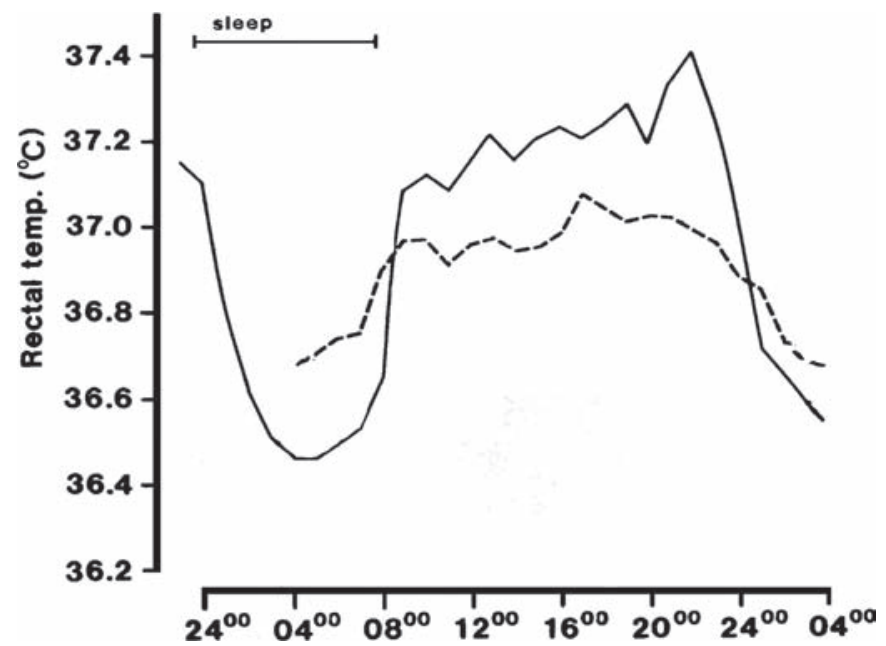

Figure 1 Mean circadian changes in rectal temperature measured hourly in eight subjects: living normally and sleeping from 24:00 to 08:00 (solid line); and then awoken at 04:00 and spending the next $24 \mathrm{~h}$ on a 'constant routine' (dashed line). Based on Minors and Waterhouse. ${ }^{64}$ only (the body clock), the exogenous component only (the individual's environment and lifestyle) or some mixture of these components. For example, Roky et al ${ }^{1}$ recorded reduced amplitudes and phase shifts in rhythms of body temperature, cortisol and melatonin during Ramadan and concluded that major chronobiological and behavioural changes occurred during fasting. This is undoubtedly true, but special chronobiological protocols are required to establish the relative roles played by the endogenous and exogenous components of each rhythm. These protocols have been described in detail ${ }^{18}$ but have not been applied at all fully in a sporting contest, and certainly not to individuals fasting in Ramadan.

\section{CIRCADIAN CHANGES IN PERFORMANCE MEASURES}

The exact cause of any measured changes to circadian rhythms in Ramadan (see below) is likely to be unknown, even though reasonable hypotheses can be suggested, of course. Before considering the effects of Ramadan upon rhythms of performance, it is necessary to be aware of circadian changes in performance that exist under normal circumstances.

\section{Physical performance}

There is fairly comprehensive evidence of circadian rhythms in many aspects of human performance, including athletic performance. ${ }^{33}$ Many of these rhythms are parallel to the circadian variation in body temperature, peaking in the late afternoon, and a causal link has been suggested. Other performance rhythms (single 'explosive' maximal efforts and actions requiring high levels of motor coordination) tend to peak earlier, around noon and early afternoon. Since peak epinephrine levels are found around noon, links with this rhythm have been proposed. By contrast, for performance with a high degree of sensorimotor coordination (eg, aiming tasks and movements requiring fine balance), the earlier phase probably reflects the circadian rhythms of cognitive performance (see below). This balance between the physical and control elements of a task is illustrated by the timing of the rhythms of the first and second serves in tennis and badminton. ${ }^{34} 35$ In both games, the first serve stresses muscle power and is observed to peak later in the day (at about the time of peak body temperature) than does performance at the second serve, which stresses placement and neuro-muscular coordination.

\section{Mental performance}

The timing of rhythms in mental performance depends upon the type of task that is being undertaken. ${ }^{36}$ For simple tasks (ie, tasks involving little cognitive processing, such as simple reaction time) the rhythm is timed similarly to that of core temperature. This is generally attributed to the general observation that neural activity increases with temperature, at least in the physiological range. For more complex tasks (eg, those requiring substantial cognitive or neuro-muscular coordination, such as decision-taking and tracking objects on a computer display screen), the time of peak of the rhythm is earlier, around midday or early afternoon. This earlier timing applies also to subjective assessments of mood, for example. These differences in timing can be understood in terms of the relative importance of 'fatigue'; in this context, fatigue does not mean muscle fatigue but a deterioration that accumulates with time awake. Such deterioration is reversed by sleep, and so the concepts of a homeostatic role for sleep and the 'sleep homeostat' have arisen. ${ }^{37}$

This interaction between time awake and the circadian component of mental performance (parallel to the rhythm of core temperature) has been modelled for alertness, ${ }^{37}$ and the general 
concepts apply to mental performance in general. Accordingly, during the morning, an individual's mental ability increases with the rise in core temperature, the effect of time awake being minimal; in the afternoon, the high core temperature offsets the increasing effect of time awake, the result of which is a plateau of performance; in the evening, performance falls due to the declining core temperature coupled with the effects of increased time awake. It can be seen that this model explains why mental performance tasks with small effects of fatigue, will be more closely in phase with the rhythm of core temperature and that more complex tasks, which are more susceptible to the effects of fatigue, will decline sooner in the daytime and show circadian rhythms that are phased earlier.

Another important factor affecting mental performance is sleep loss, and loss of as little as $2 \mathrm{~h}$ of sleep can cause a decrement. ${ }^{38}$ Sleep loss need not be associated with a single night; it could be cumulative-say, $0.5 \mathrm{~h}$ per night for four successive nights. The most obvious effect of sleep loss is the subjective one of daytime fatigue, but objective tests of performance show falls also. Small sleeps (naps) in the daytime can alleviate the effects of sleep loss, the total amount of sleep obtained per $24 \mathrm{~h}$ rather than its distribution appearing to be the important factor. However, there are two caveats to this last statement. First, immediately after any sleep or nap, there is a temporary period of decreased mental ability as the subject wakes up fully; this decrement is attributed to 'sleep inertia.' 39 Second, naps substantially shorter than $1 \mathrm{~h}$ provide insufficient time for the slow wave stages of sleep, the type of sleep that has most recuperative value, to be reached. ${ }^{40}$

\section{TRAINING}

Given that circadian rhythms are ubiquitous, it is not surprising that their effects impinge upon training schedules.

\section{Time of training in normal circumstances}

The timing of training sessions often depends upon the individual's lifestyle, training occurring at lunchtime or before or after work. However, the effectiveness of training depends upon the amount of work that is done. This is important because of an individual's perceived exertion which shows a circadian rhythm that is in phase with core temperature. This means that more work can normally be tolerated in the late afternoon than at other times of the day ${ }^{33}$ and that a given amount of work is perceived as being least hard at this time. However, there is some suggestion of habituating to training at a particular time of day, Edwards et $a^{41}$ reporting that competitive performance in cycle time-trials is influenced positively by training at that time in the days before racing.

Training in the afternoon has advantages, therefore, and there are other reasons why other times might be non-ideal. Early in the morning, soon after waking, the intervertebral discs of the spinal cord are slightly swollen due to absorb fluid during sleep when the spine is unloaded. Such swelling might increase the risk of damage (a 'slipped disc') until this excess fluid has exuded from the discs, normally about $1-2 \mathrm{~h}$ after becoming upright. ${ }^{29}$ In addition, the cardiovascular response to exercise is greater in the morning than later in the day, a set amount of exercise causing a greater rise of blood pressure. ${ }^{42}$ While the possible damage this might cause has not been assessed epidemiologically, it coincides with the time of the highest frequency of cardiovascular morbidity from ischaemia and haemorrhage, ${ }^{43} 44$ and so caution is advised. If such rhythms in morbidity have an exogenous component (and this is not known), training immediately after a siesta would also be advised against.
In the evening, training sessions are likely to impede sleep onset due to the raised core temperature they produce.

\section{Training in Ramadan}

For individuals training during Ramadan, there will be several differences from normal-altered times of food and fluid intake (dealt with in other articles), increased fatigue due to loss of nocturnal sleep and increased time awake (due to rising early). Many of these changes will affect measured circadian rhythms.

\section{Altered circadian rhythms}

The rhythms of cortisol and testosterone normally display a peak in the morning around the time of awakening. Bogdan et $a l^{45}$ reported a shift of secretion onset in both of these hormones as well as an altered profile of cortisol secretion, with reduced secretion in the morning and increased secretion in the evening. Thus, in control conditions, values at 08:00 were 3.84 times higher than at 20:00 h, a difference that was reduced to 2.02 times during Ramadan. They also observed an enhanced peak in prolactin, and a diminished and delayed peak in melatonin, secretion, findings consistent with increased fatigue and retiring later. The confinement of eating to the early and late parts of the day also delayed the time of peak levels of serum leptin by more than 5 h. ${ }^{46}$ Roky et al ${ }^{47}$ reported a diurnal decrease in oral temperature between 9:00 and 20:00 and an increase at 23:00 and 24:00 during Ramadan, indicative of a decreased amplitude of the rhythm.

Bahammam ${ }^{15}$ reported delayed bedtime and waking time in fasting subjects during Ramadan and an increased tendency towards 'eveningness' behaviour. The tendency to delay going to sleep and increase evening activities (often with a strong social component) has been reported by others, and is often accompanied by late waking and increased daytime sleepiness. ${ }^{48}$ In a study in the United Arab Emirates, Margolis and Reed $^{16}$ reported that Muslims avoided a rise in daytime somnolence by increasing daytime sleep hours during Ramadan. Roky et $a l^{17}$ allowed Muslim subjects an opportunity for daytime naps and observed reduced sleep latency during them, indicating fatigue. They also observed polysomnographically the nocturnal sleep of Muslims during Ramadan, finding increased sleep latency and modified sleep architecture. ${ }^{49}$ The total sleep time decreased, but the proportion of non-REM sleep increased, with increased stage 2 sleep and decreased SWS.

\section{Possible causes of the altered rhythms}

The detailed causes of such changes cannot be deduced from these observations, and complex protocols are required to do so. ${ }^{18}$ In practice, therefore, such information is lacking. Nevertheless, changes in the secretion of hormones associated with food intake and metabolism will be at least partly due to altered mealtimes and sleep-wake cycles; mental performance will be compromised due to partial sleep loss and to rising earlier; and gut function (see section 'Effects of Changed Food Intake') will alter due to changed meal times and type of food eaten.

Subjective estimates of physical activity during control days and Ramadan in subjects not undertaking training regimens are illustrated in figure $2 .{ }^{50}$ Fasting subjects 'spare' themselves during the fast time (daytime) and become more active once the fast has been broken (evening). Such changes also apply estimates of mental and social activities and the amounts of physical and mental activity that are wished for. In addition, increased fatigue will negatively affect motivation at all times. This change, together with the flattening of the rhythms (figure 2), will translate into decreased motivation to train effectively in the daytime. 
It is possible that the phase of the body clock will change in so far as the zeitgebers have changed. Rising earlier in the morning will expose the individual to artificial light early in the day, and even though it is less effective than sunlight in shifting the body clock, it will tend to advance the body clock (directly and by suppressing melatonin secretion); by contrast, extended light exposure in the evening will tend to delay the body clock. Therefore, the direction or size of shift produced by the changed light exposure cannot be predicted. Increased physical activity in the evening is likely to produce a small delay in the body clock.

\section{EFFECTS OF CHANGED FOOD INTAKE}

The alterations of food intake patterns will have a marked effect upon many aspects of gastrointestinal activity, since these have large exogenous components. In addition, the large meal in the evening is likely to hinder sleep onset and upset the normal balance between daytime metabolism of glucose and nocturnal metabolism of fat from adipose tissue.

Even though mealtimes have only a weak role as a possible zeitgeber (above), the type of food eaten might exert an effect. There is evidence that monoamines, principally serotonin, modulate both sleep and metabolism. Dietary tryptophan is the biochemical precursor of serotonin, and plasma tryptophan rises after ingesting carbohydrate; therefore, it has been argued that a high-carbohydrate meal would induce drowsiness and promote sleep. ${ }^{51}$ However, only minute changes in the EEG have been reported in athletes using a high-carbohydrate diet. ${ }^{52}$ Also, it has not been firmly established that precursor uptake is the rate-limiting step for serotonin release, or that such changes are responsible for the fall in alertness. ${ }^{53}$ Nevertheless, MinetRinguet et $a l^{54}$ reported that a protein diet rich in tryptophan restored sleep after food deprivation in the rat. Serotonin may have a role also in regulating the secretion of insulin by the pancreas; inhibition of serotonin decreases insulin secretion, ${ }^{55}$ and sleep is reduced when subjects are deprived of food. This implies that fasting during Ramadan would render sleep more difficult in the daytime (lower insulin levels), but this will be offset by the decreased amount of nocturnal sleep. ${ }^{56}$

Tyrosine is the precursor of catecholamines, and it is known that a high-protein meal increases plasma tyrosine and its uptake into the brain. It has been argued that this would promote the synthesis and release of catecholamines, increasing alertness. ${ }^{51}$ Again, it is not firmly established that precursor uptake is the rate-limiting step for catecholamine release. Nevertheless, it remains possible that appropriate changes to the diet could be used to improve alertness and sleep during Ramadan.

\section{Gastrointestinal function}

The question arises as to whether eating in Ramadan at times that differ from non-fasting days places undue strain on the neuromuscular activity of the gastrointestinal tract and its secretions or upon the absorption and metabolism of digested foodstuffs. The issue becomes one of considering the relative size of the endogenous and exogenous components of these functions. If the exogenous component is strong, then altered food intake will be reflected in equal changes in timing of gastrointestinal function; by contrast, if the endogenous component is marked, the clock-driven processes will function less efficiently at the 'wrong' times of day-early in the morning or late in the evening or at night, for example-and gastrointestinal function will be poorer and placed under strain. This problem is observed in night workers when eating a meal at about 02:00 in the middle of their shift. A lack of interest in food, indigestion and an increased incidence of ulcers are widely
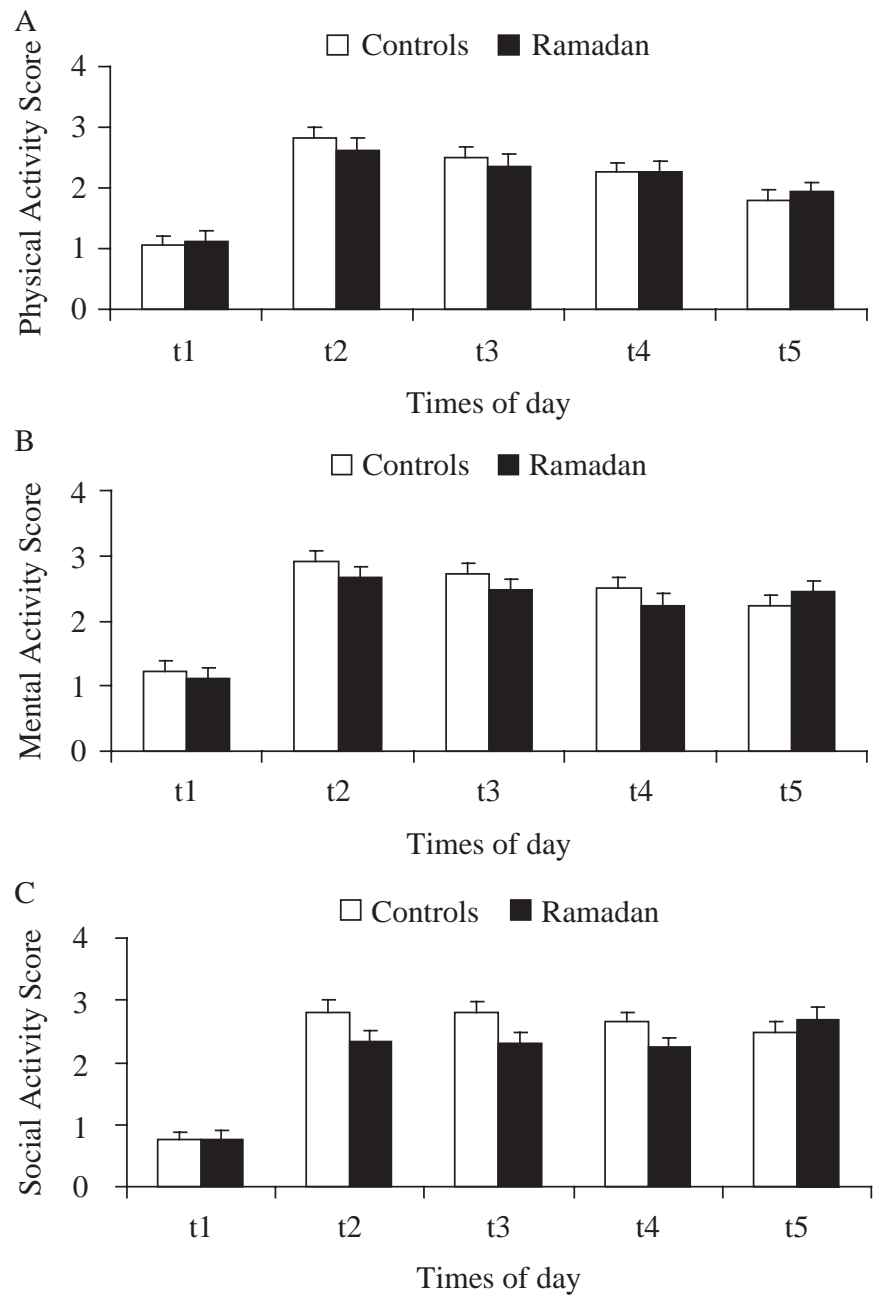

Figure 2 Scores for (A) physical activity; (B) mental activity; and (C) social activity during control days and Ramadan. Mean+SE shown. Scores range from 0 , 'not at all' to 4 , 'very much.' $\mathrm{t} 1=$ rising-10:00; $\mathrm{t2}=10: 00-13: 00 ; \mathrm{t} 3=13: 00-16: 00 ; \mathrm{t} 4=16: 00-$ $19: 00 ; t 5=19: 00-22: 00$, sunrise being at 07:00 and sunset at 19:00. Based on Waterhouse et al. ${ }^{50}$

reported in this group. ${ }^{57}$ There are several reasons for such problems, but an inappropriately timed body clock (adjusting to night work slowly and incompletely) is one of them.

The normal pattern of eating is reflected in similarly timed rhythms of gut motility, secretion of digestive juices, absorption of digested food, blood concentrations of glucose, amino acids and lipids. ${ }^{58}$ This indicates a large exogenous component of the rhythms, but there is also evidence for an endogenous component also, since the gastrointestinal and metabolic effects produced by identical meals display circadian variation; metabolic responses to a glucose load, for example, are less rapid in the evening than in the morning. ${ }^{59}$ Moreover, gastric emptying and blood flow are greater in the daytime than at night, leading to a faster absorption of foodstuffs from the gastrointestinal tract; ${ }^{60}$ by contrast, the absorption of some drugs from the gastrointestinal tract is greater in the hours after waking due to an empty stomach.

A further indication that gut function shows a circadian rhythm with an endogenous component comes from investigations of hunger and the incidence of indigestion in a group of subjects undergoing a constant routine. The constant routine lasted $25 \mathrm{~h}$ and began at 08:00 (figure 3 ). At the end of each hour 
awake, the subjects were asked to rate their hunger (on a scale from -5 , not at all hungry, to 0 , as hungry as normal, to +5 , very hungry). They were also asked to rate any feelings of indigestion from 0 (none) to +5 (marked). During their normal waking hours (the first $16 \mathrm{~h}$ of the constant routine) their hunger was normal, and they suffered little from indigestion. However, during the night, their hunger fell, and the incidence of indigestion increased, indicating that the gut absorbs food less well at night. It has a direct relevance to Muslims, who have eaten a large meal after sunset; they will feel bloated, and this will hinder sleep.

When the metabolism of absorbed foodstuffs is considered, several hormones (eg, insulin, ghrelin, leptin, growth hormone, glucagon and cortisol) are involved. As indicated above, the secretion profiles of these change during Ramadan, and at least for some (eg, insulin and cortisol), there is evidence that the rhythms possess an endogenous component. In such cases, secretion will be predicted not to change fully in accordance with altered mealtimes, since the body clock will not have adjusted fully to the changed habits.

Clinically, there are potential consequences of increased gastric acidity during fasting in individuals with duodenal ulcers. Iraki et al considered that patients healed of this condition had an increased risk of relapse due to a continuously low gastric $\mathrm{pH}$ and high plasma gastrin concentrations during the hours of fasting. ${ }^{61}$ Also, eating particular types of food at inappropriate times may be a factor in gastrointestinal complaints among those on unorthodox daily work-schedules and may predispose individuals to becoming overweight, not unlike the position in night workers. ${ }^{57}$ However, Iraki et al ${ }^{62}$ also showed that gastric acidity was increased during Ramadan, mainly in the diurnal phase, but without any adverse digestive symptoms.

\section{CHANGING THE TIME OF TRAINING}

Normally, training sessions occur during the daytime, for reasons of convenience. This time is also advantageous because that value of training depends upon the amount of work performed, and this is greatest around the time of peak of core temperature in the late afternoon. Training at other times, early or late in the day, is likely to be less effective; not only will the
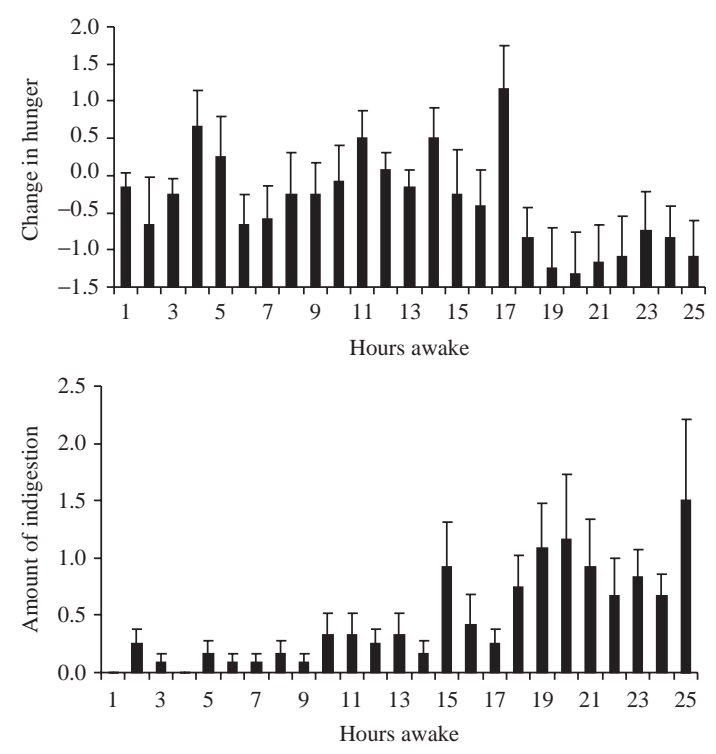

Figure 3 Changes from normal days in hunger, top, and amount of indigestion, bottom, in a group of 12 subjects who underwent a $25 \mathrm{~h}$ constant routine. Mean+SE shown. For more details, see text. time of training be before (early morning) or after (evening) the times of peak physical and mental performance, but also any work load will be perceived as being harder. ${ }^{33}$ Training during Ramadan is often postponed until after sunset; ${ }^{4}$ nevertheless, even if the exercise intensity is lower when taken earlier or later in the day than is customary, the practice of game skills will have obvious value over the total exclusion of training.

There is evidence that habituation to training at a particular time of day occurs in cycle time-trials, Edwards et al ${ }^{41}$ reporting that competitive performance in cycle time-trials was influenced positively by training at that time in the days before racing. While exercising in the daytime during Ramadan may lead to such a habituation effect, whether it applies also when training is delayed until after sunset is unknown. Training in the morning 2-3 h after the presunrise meal ('scoor') leads to fluid and energy losses, and there is no opportunity for their rapid restoration following cessation of activity as normally occurs. The alternative, to train after sunset, is practised more frequently. The first intake of solids after sunset ('footor') is typically dates taken with water, and this will replenish some of the fluid and calorie deficits accumulated during fasting. Absorption could take place after footor and prayers but before the major meal, and exercise training could be performed in this interval. There might also be benefits to exercising after darkness has fallen when, in hot climates, the high environmental outdoor temperatures are avoided.

Evening training might delay the body clock. ${ }^{32}$ If this were so, training would be taking place closer to the times of peak of performance, and this would increase the work that could be done, the motivation to perform maximally and the value of the training. However, delaying the body clock in this way has negative consequences; the abilities to get to sleep and to wake in the morning would be delayed also. Some studies indicate that bedtime and rising times are substantially delayed in Ramadan, individuals rising quite late in the morning, and training performance after sunset is maintained. ${ }^{4}$ However, no information relating to the phase of the body clock in such circumstances is available.

It must also be remembered that, even though adopting such training regimens might be acceptable in Muslim countries, they are likely to be impossible in other countries-in Europe, for example, where training takes place in the daytime.

\section{Conclusions on changed habits during Ramadan}

Ramadan imposes particular demands upon training athletes. These demands arise from not only the lack of food and fluid intake but also sleep loss. There is a tendency to delay training sessions until after sunset and following ending the fast. These changes will alter the normal circadian rhythms of performance and so have something in common with those seen after a timezone transition, during night work or in ageing; however, in detail, they are unique to Ramadan, and so no clear model exists for predicting them in any detail. ${ }^{63}$ Circadian rhythms of performance, hormones and gastrointestinal function alter in response to the changed hours of sleep, and food and fluid intake, but current data do not enable changes to be attributed to an altered body clock, to differences in the individual's lifestyle and environment or to both factors. Further research is needed to clarify this issue. Changed times of training are unlikely to occur in non-Muslim countries, and daytime training in Ramadan is likely to result in a decreased value of the training regimen.

Competing interest None.

Provenance and peer review Commissioned; externally peer reviewed. 


\section{REFERENCES}

1. Roky R, Houti I, Moussamih S, et al. Physiological and chronobiological changes during Ramadan intermittent fasting. Ann Nutr Metab 2004;48:296-303.

2. Kadri N, Tilane A, El Batal M, et al. Irritability during the month of Ramadan. Psychosom Med 2000;62:280-5.

3. Ennigrou S, Zenaidi M, Ben Slama F, et al. Ramadan and customs of life: investigation with 84 adult residents in the district of Tunis. Tunis Med 2001;79:508-14

4. Wilson D, Drust B, Reilly T. Is diurnal lifestyle altered during Ramadan in professional Muslim athletes? Biol Rhythms Res 2009;40:385-97.

5. Meckel Y, Ismaeel A, Eliakim A. The effect of the Ramadan fast on physical performance and dietary habits in adolescent soccer players. Eur J Appl Physiol 2008;102:651-7.

6. Stannard SR, Thompson MW. The effect of participation in Ramadan on substrate selection during submaximal cycling exercise. J Sci Med Sport 2008;11:510-17.

7. Kirkendall DT, Leiper JB, Bartagi Z, et al. The influence of Ramadan on physical performance measures in young Muslim footballers. J Sports Sci 2008;26(Suppl 3):S15-27.

8. Leiper JB, Watson P, Evans G, et al. Intensity of a training session during Ramadan in fasting and non-fasting Tunisian youth football players. J Sports Sci 2008;26(Suppl 3):S71-9.

9. Zerguini Y, Kirkendall D, Junge A, et al. Impact of Ramadan on physical performance in professional soccer players. Br J Sports Med 2007:41:398-400.

10. Zerguini Y, Dvorak J, Maughan R, et al. Influence of Ramadan fasting on physiological and performance variables in football players: summary of the F-MARC 2006 Ramadan fasting study. J Sports Sci 2008;26:S3-6.

11. Leiper JB, Junge A, Maughan RJ, et al. Alteration of subjective feelings in football players undertaking their usual training and match schedule during the Ramadan fast. J Sports Sci 2008;26(Suppl 3):S55-69.

12. Ramadan J. Does fasting during Ramadan alter body composition, blood constituents and physical performance? Med Prin Pract 2002;11:41-6.

13. Bigard AX, Boussif M, Chalabi $\mathrm{H}$, et al. Alterations in muscular performance and orthostatic tolerance during Ramadan. Aviat Space Environ Med 1998; 69:341-6

14. Siddiqui $\mathbf{0 A}$, Sabir S, Subhan MM. The effect of Ramadan fasting on spirometry in healthy subjects. Respirology 2005;10:525-8.

15. BaHammam A. Assessment of sleep patterns, daytime sleepiness, and chronotype during Ramadan in fasting and nonfasting individuals. Saudi Med J 2005;26:616-22.

16. Margolis SA, Reed RL. Effect of religious practices of Ramadan on sleep and perceived sleepiness of medical students. Teach Learn Med 2004;16:145-9.

17. Roky R, Chapotot F, Benchekroun MT, et al. Daytime sleepiness during Ramadan intermittent fasting: polysomnographic and quantitative waking EEG study. J Sleep Res 2003;12:95-101.

18. Reilly T, Waterhouse J. Sports performance: is there evidence that the body clock plays a role? Eur J Appl Physio/ 2009;106:321-32.

19. Bellisle F, McDevitt R, Prentice AM. Meal frequency and energy balance. Br J Nutr 1997;77(Suppl 1):S57-70.

20. Chaouachi A, Chamari K, Roky R, et al. Lipid profiles of judo athletes during Ramadan. Int J Sports Med 2008;29:282-8.

21. Bouhlel E, Denguezli M, Zaouali M, et al. Ramadan fastings effect on plasma leptin, adiponectin concentrations, and body composition in trained young men. Int J Sport Nutr Exerc Metab 2008;18:617-27.

22. Bouhlel E, Zaouali M, Miled A, et al. Ramadan fasting and the GH/IGF-1 axis of trained men during submaximal exercise. Ann Nutr Metab 2008;52:261-6.

23. Leiper JB, Maughan RJ, Kirkendall DT, et al. The F-MARC study on Ramadan and football: research design, population, and environmental conditions. J Sports Sci 2008;26(Suppl 3):S7-13.

24. Maughan RJ, Bartagi Z, Dvorak J, et al. Dietary intake and body composition of football players during the holy month of Ramadan. J Sports Sci 2008;26(Suppl 3):S29-38.

25. Maughan RJ, Leiper JB, Bartagi Z, et al. Effect of Ramadan fasting on some biochemical and haematological parameters in Tunisian youth soccer players undertaking their usual training and competition schedule. J Sports Sci 2008;26(Suppl 3):S39-46.

26. Shirreffs SM, Maughan RJ. Water and salt balance in young male football players in training during the holy month of Ramadan. J Sports Sci 2008;26(Suppl 3):S47-54.

27. Bouhlel E, Salhi Z, Bouhlel H, et al. Effect of Ramadan fasting on fuel oxidation during exercise in trained male rugby players. Diabetes Metab 2006;32:617-24.

28. Ramadan J, Telahoun G, Al-Zaid NS, et al. Responses to exercise, fluid, and energy balances during Ramadan in sedentary and active males. Nutrition 1999;15:735-9.

29. Reilly T, Atkinson G, Waterhouse J. Biological rhythms and exercise. Oxford: Oxford University Press, 1997

30. Atkinson G, Drust B, Reilly T, et al. The relevance of melatonin to sports medicine and science. Sports Med 2003;33:809-31.

31. Waterhouse J, Reilly T, Atkinson G, et al. Jet lag: trends and coping strategies. Lancet 2007;369:1117-29.
32. Atkinson G, Edwards B, Reilly $\mathrm{T}$, et al. Exercise as a synchroniser of human circadian rhythms: an update and discussion of the methodological problems. Eur J App/ Physio/ 2007:99:331-41.

33. Atkinson G, Reilly T. Circadian variation in sports performance. Sports Med 1996;21:292-312.

34. Atkinson G, Speirs L. Diurnal variation in tennis service. Percept Mot Skills 1998:86:1335-8.

35. Edwards $\mathbf{B J}$, Lindsay K, Waterhouse J. Effect of time of day on the accuracy and consistency of the badminton serve. Ergonomics 2005;48:1488-98.

36. Waterhouse J, Minors D, Akerstedt T, et al. Rhythms of human performance. In: Takahashi J, Turek F, Moore F, eds. Handbook of behavioral neurobiology: circadian clocks. New York: Kluver Academic/Plenum Publishers, 2001:571-601.

37. Akerstedt $\mathbf{T}$, Folkard $\mathrm{S}$. Validation of the $\mathrm{S}$ and $\mathrm{C}$ components of the threeprocess model of alertness regulation. Sleep 1995;18:1-6.

38. Akerstedt T. Altered sleep/wake patterns and mental performance. Physiol Behav 2007:90:209-18.

39. Naitoh P, Kelly T, Babkoff H. Sleep inertia: best time not to wake up? Chronobiol Int 1993;10:109-18.

40. Dinges D. Adult napping and its effect on ability to function. In: Stampi C, ed. Why we nap. Massachusetts, Boston, USA: Birkhauser, 1992:118-134.

41. Edwards BJ, Edwards W, Waterhouse J, et al. Can cycling performance in an early morning, laboratory-based cycle time-trial be improved by morning exercise the day before? Int J Sports Med 2005;26:651-6.

42. Jones H, Atkinson G, Leary A, et al. Reactivity of ambulatory blood pressure to physical activity varies with time of day. Hypertension 2006;47:778-84.

43. Portaluppi F, Manfredini R, Fersini C. From a static to a dynamic concept of risk: the circadian epidemiology of cardiovascular events. Chronobiol Int 1999;16:33-49.

44. Smolensky M, Hermida R, Portaluppi F, et al. Twenty-four-hour pattern of angina pectoris, myocardial infarction, and sudden cardiac death: Potential role of blood pressure, heart rate and rate-pressure product. Biol Rhythm Res 2007; 38: 205-16.

45. Bogdan A, Bouchareb B, Touitou Y. Ramadan fasting alters endocrine and neuroendocrine circadian patterns. Meal-time as a synchronizer in humans? Life Sci 2001;68:1607-15

46. Bogdan A, Bouchareb B, Touitou Y. Response of circulating leptin to Ramadan daytime fasting: a circadian study. Br J Nutr 2005;93:515-18.

47. Roky R, Iraki L, HajKhlifa R, et al. Daytime alertness, mood, psychomotor performances, and oral temperature during Ramadan intermittent fasting. Ann Nutr Metab 2000;44:101-7.

48. Taoudi Benchekroun M, Roky R, Toufiq J, et al. Epidemiological study: chronotype and daytime sleepiness before and during Ramadan. Therapie 1999:54:567-72.

49. Roky R, Chapotot F, Hakkou F, et al. Sleep during Ramadan intermittent fasting. J Sleep Res 2001:10:319-27.

50. Waterhouse J, Alkib L, Edwards B et al. Diurnal changes in sleep, food and fluid intakes, and activity during Ramadan, 2006, in the UK; some preliminary observations. Biol Rhythm Res 2008;39:449-67.

51. Leathwood P. Circadian rhythms of plasma amino acids, brain neurotransmitters and behaviour. In: Arendt J, Minors D, Waterhouse J, eds. Biological rhythms in clinical practice. Guildford: Butterworth, 1989:136-159.

52. Davenne D, Francart A, Ranaud, A. The effects of two types of diet on subsequent sleep. Proceedings of the 10th European Sleep Research Society Conference. Stuttgart, Germany: Gustav Fischer Verlag, 2005:106.

53. Waterhouse J, Reilly T, Atkinson G. Jet-lag. Lancet 1997;350:1611-16.

54. Minet-Ringuet J, Le Ruyet PM, Tomé D, et al. A tryptophan-rich protein diet efficiently restores sleep after food deprivation in the rat. Behav Brain Res 2004; 152:335-40.

55. Bird JL, Wright EE, Feldman JM. Pancreatic islets: a tissue rich in serotonin. Diabetes 1980;29:304-8.

56. Dement WC. Sleep extension: getting as much extra sleep as possible. Clin Sports Med 2005:24:251-68, viii.

57. Waterhouse J, Folkard S, Minors D. Shiftwork, health and safety. an overview of the scientific literature 1978-1990. Report for Health and Safety Executive. London: HMSO, 1991

58. Mejean L, Bicakova-Rocher A, Kolopp M, et al. Circadian and ultradian rhythms in blood glucose and plasma insulin of healthy adults. Chronobiol Int 1988;5:227-36.

59. Wolever, T Bolognesi, C. Time of day influences relative glycaemic effect of foods. Nutr Res 1996, 16: 381-4.

60. Sanders SW, Moore JG. Gastrointestinal chronopharmacology: physiology, pharmacology and therapeutic implications. Pharmacol Ther 1992:54:1-15.

61. Iraki L, Bogdan A, Hakkou F, et al. Ramadan diet restrictions modify the circadian time structure in humans. A study on plasma gastrin, insulin, glucose, and calcium and on gastric pH. J Clin Endocrinol Metab 1997:82:1261-73.

62. Iraki L, Abkari A, Vallot T, et al. Effect of Ramadan fasting on intragastric $\mathrm{pH}$ recorded during 24 hours in healthy subjects. Gastroenterol Clin Biol 1997;21:813-19.

63. Reilly T, Waterhouse J. Altered sleep-wake cycles and food intake: the Ramadan model. Physiol Behav 2007:90:219-28.

64. Minors D, Waterhouse J. Circadian rhythms and the human. Bristol: John Wright, 1981. 